A LETTERS JOURNAL EXPLORING THE FRONTIERS OF PHYSICS

\title{
OFFPRINT
}

\section{Grain size effects on lateral islands in hard-sphere crystals}

V. W. A. De Villeneuve, P. S. Miedema, J. M. Meijer and A. V. Petukhov

EPL, 79 (2007) 56001

Please visit the new website www.epljournal.org 


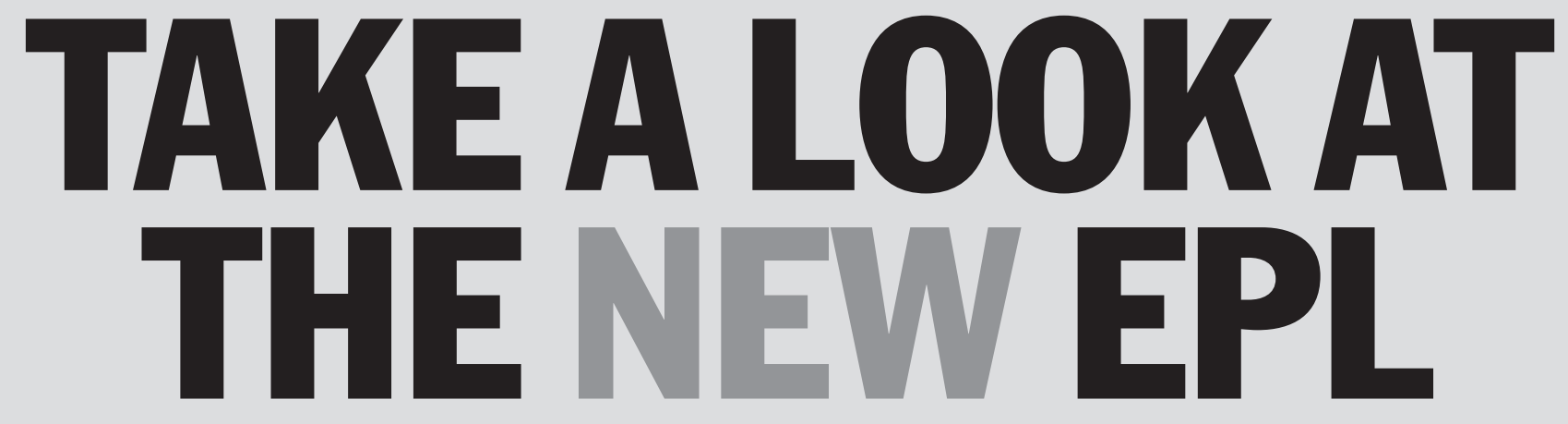

\section{Europhysics Letters (EPL) has a new online home at www.epljournal.org}

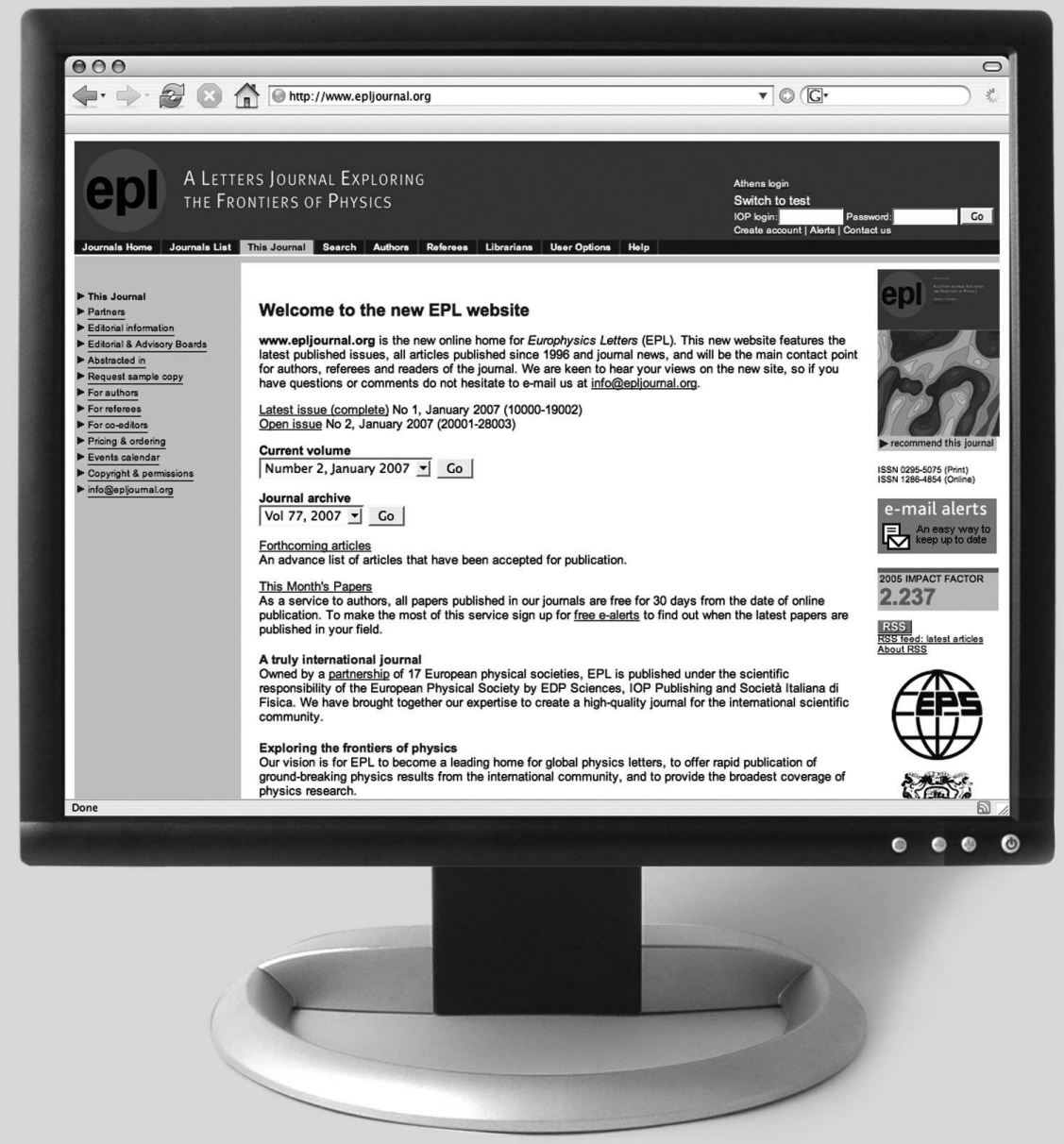

Take a look for the latest journal news and information on:

- reading the latest articles, free!

- receiving free e-mail alerts

- submitting your work to EPL 


\title{
Grain size effects on lateral islands in hard-sphere crystals
}

\author{
V. W. A. de Villeneuve, P. S. Miedema, J. M. Meijer and A. V. Petukhov \\ Van 't Hoff Laboratory for Physical and Colloid Chemistry, University of Utrecht - Padualaan 8, 3584 CH Utrecht, \\ The Netherlands
}

received 10 April 2007; accepted in final form 5 July 2007

published online 31 July 2007
PACS 61.72.Ff - Direct observation of dislocations and other defects (etch pits, decoration, electron microscopy, X-ray topography, etc.)
PACS 82.70.Dd - Colloids
PACS 87.64.Tt - Confocal microscopy

\begin{abstract}
Due to the lateral stacking disorder, random hexagonally closed-packed hard-sphere crystals consist of lateral islands with different lateral positions $\mathrm{A}, \mathrm{B}$, and $\mathrm{C}$, and as a consequence, different stacking. We investigate the extent of lateral stacking disorder as a function of grain size, and as a function of the fraction of FCC-stacked particles $\alpha$ by laser scanning confocal microscopy and Monte Carlo simulations. We compare the simulations and microscopy data to relate stacking islands (2D domains with identical stacking type and direction) to lateral islands. Small crystals mainly contain single hexagonal planes, whereas larger crystals consist of a much larger number of lateral islands. Furthermore, the typical stacking island size is related to the FCC fraction. At high $\alpha$, more FCC islands nucleate, and these are more likely to combine into larger islands than the HCP islands.
\end{abstract}

Copyright (c) EPLA, 2007

Introduction. - Small crystallites are remarkably different from their large counterparts. The amount of particles at the surface is relatively high, which can drastically affect elastic [1], optical [2], electric [3] and magnetic properties [4]. The change in properties is often coupled to a change in particle configuration. It is extremely difficult to study atomic systems on the single-particle level, but colloidal systems are excellent reference systems: they are very similar to atomic systems [5] and enable $3 \mathrm{D}$ studies on the single-particle level by confocal microscopy [6-9] due to large particle size and their relative slowness. The phase diagram of colloidal hard spheres is based on excluded-volume interactions only. The free energy difference between face-centered cubic (FCC) and hexagonally closed packed (HCP) structures is tiny for hard sphere crystals [10-12]: less than $10^{-3} k_{B} T$ per particle, where $k_{B} T$ is the thermal energy. As a result, a so-called randomhexagonal close-packed (RHCP) crystal structure is often found in experimental hard sphere systems [13-18]: the reorganisation from RHCP to FCC structure is expected to take months to years for experimental systems $[18,19]$. Due to the variety of possible RHCP configurations, an additional gain in entropy can stabilize the RHCP structure for sufficiently small crystals [20]. The RHCP structure is characterized not only by randomly alternating FCC and HCP layers, but also by lateral islands with different lateral positions A, B and C [21], and as a result different stacking. Lateral islands (2D domains with identical lateral position) and stacking islands (2D domains with identical stacking) are different quantities: stacking is based on the lateral position of three layers instead of one for lateral islands. In our previous work [21] we were able to identify line-defects, but unable to identify the typical island dimensions. Here, we extend the methods introduced there to investigate the extent of island formation as a function of grain size and as a function of the fraction of FCC-stacked particles $\alpha$. We compare results obtained by confocal microscopy in sedimented hard sphere crystals with results of Monte Carlo simulations. Note that atomic systems do not grow by sedimentation, but usually by heterogeneous nucleation and growth. In both scenarios, the growth is directed, which should result in similar growth mechanisms. Colloidal systems have a higher defect density than atomic systems, but the typical grain size is usually much larger as well. In both cases the extent of island formation should be strongly dependent on the grain size.

Experimental section. - Full details of our experimental methods can be found in [21]. We prepared sedimented crystals of fluorescent, 1.2 diameter $\mu \mathrm{m}$ polymethyl-metacrylate spheres [22], dispersed in two apolar 
solvents: a mixture of tetrachloromethane, tetralin and cis-decalin (TTC) (0.35:0.35:0.30 v/v) and pure cisdecalin (De). Our previous studies showed that the different solvents resulted in fractions of FCC $\alpha$ of $\sim 0.6$ (TTC) and $\sim 0.7$ (De) [21]. Crystals were grown from the sample bottom by sedimentation from intial volume fractions $\phi_{0}$ of 0.4 (Sample TTC6) and 0.2 (Sample De10), 0.3 (De13) and 0.4 (De14). Thin 2D cross-sections of the sample were imaged with a Nikon Eclipse TE2000U laser scanning confocal microscope with a Nikon $\mathrm{C} 1$ scanning head in combination with an argon laser $\left(\lambda_{0}=488 \mathrm{~nm}\right)$ and an oil-immersion lens (Nikon Plan APC 100X, NA 1.4). The crystals' hexagonal layers were imaged from the glass sample bottom to sample heights of $\sim 30 \mu \mathrm{m}$, which corresponds to the first 20-30 layers of a crystal. Images were taken of complete grains (De10, De14), as well as within grains (De13, TTC6).

Data analysis. - Particle positions were obtained by methods such as those used by Crocker and Grier [23]. We will now shortly explain how stacking islands were identified, and how particles within a single grain were isolated using IDL routines ${ }^{1}$.

Identification of islands. Within a crystal layer, several islands with different lateral positions A, B and $\mathrm{C}$ can be present, as for example in the confocal image shown in fig. 1A. Fitting such a layer to a grid of A, B and $\mathrm{C}$ positions is not straightforward. Although some lateral islands can be distinguished, as shown in fig. 1B, over longer distance the method fails due to dislocations and slow lattice deformations [24]. The polydispersity of the system likely enhances these effects [19]. The changes in lateral positions can roughly be divided into abrupt changes and slow changes in lateral position. The abrupt changes are characterised by line-defects which form the boundary between two lattice positions, such as illustrated in fig. 1C. The slow changes are characterised by partial dislocations such as illustrated by the partial screw dislocation in fig. 1D. Three consecutive layers are shown there, which show a marked transition of ABA stacking (left) to ABC stacking (right). The screw dislocation is characterised by a Burger vector with a direction parallel to the lattice deformation (the in and out of plane vectors between the first and the third plane are not shown). In practice, the two can occur simultaneously, as for example in the left bottom corner of fig. 1A, where the line-defect slowly "disappears" in the lattice. The identification of lateral islands is therefore not feasible in large real-space datasets on the basis of criteria used in [21]. A change in lateral position is usually coupled to a change in stacking (HCP or FCC). Stacking can subsequently be characterised to distinguish islands, but since stacking across both sides of a line-defect can be HCP and FCC on both sides, a second criterium is necessary. Here we extend the use of

\footnotetext{
${ }^{1}$ Interactive data language, research systems, inc. (http:// www.ittvis.com/).
}
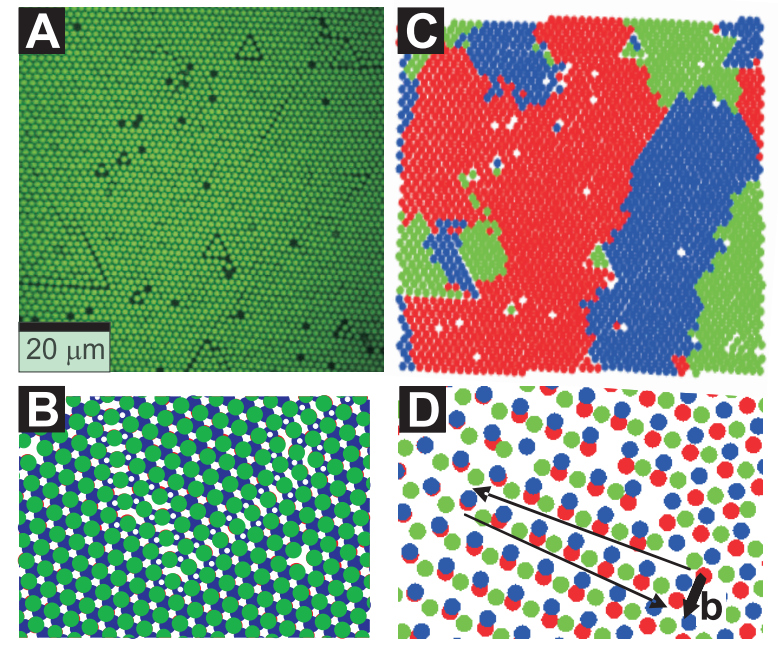

Fig. 1: (Colour on-line) Lateral stacking disorder in hard-sphere crystals. A) A typical confocal image of a close-packed crystal plane. B) A fit of the particle positions to an ideal lattice of $\mathrm{A}, \mathrm{B}$ and $\mathrm{C}$ positions. Three consecutive layers (red, blue, green circles) are shown in (C) and (D). In (C) two HCP domains are separated by a clearly visible line-defect (marked by open circles). In (D) a partial screw dislocation (with Burgers vector $\mathbf{b}$, the in- and out of plane vectors are not shown) causes a slow transitions from one lateral position to the other. The particles in the middle layer slowly shift from ABA to ABC environment.

the orientational correlation method presented in [21] by incorporating the stacking direction.

The orientational correlation method compares the particle orientations of the neighbours of a particle $j$. All particles $j$ in layer $L$ are projected onto the layers $L+1$ (above) and $L-1$ (below), and next neighbours are identified. The relative rotation between the particles in layers $L+1$ and $L-1$ is $60^{\circ}$ for $\mathrm{FCC}$ and $0^{\circ}$ for $\mathrm{HCP}$ (fig. 2A). To express this rotation the bond orientational order parameter $\psi_{3}^{ \pm}$is introduced:

$$
\psi_{3}^{ \pm}\left(\mathbf{r}_{j}\right)=\frac{1}{N^{ \pm}} \sum_{k^{ \pm}}^{N^{ \pm}} \exp \left[3 i \theta\left(\mathbf{r}_{j k^{ \pm}}\right)\right] .
$$

The summation $k^{ \pm}$runs over all $N^{ \pm}$next neighbours in the layer above $\left(\psi_{3}^{+}\right)$or below $\left(\psi_{3}^{-}\right)$of a given particle $j$. The angle between the bond vector connecting the particle with next neighbour $k^{ \pm}$and a fixed arbitrary reference axis is defined as $\theta_{\mathbf{r}_{\mathbf{j k}}}$. As a result, stacking can now easily be assigned: $\left|\psi_{3}^{+}+\psi_{3}^{-}\right| \simeq 0$ and 2 for FCC and HCP, respectively.

Figure 2B shows that besides a stacking type, a stacking direction can be distinguished as well. For HCP this comes down to stacking of type $\mathrm{HCP}_{1}$ of $\mathrm{ABA}$, and stacking of type $\mathrm{HCP}_{2}$ of $\mathrm{BAB}$. Similarly, for FCC this results in stacking of type $\mathrm{FCC}_{1}$ of $\mathrm{ABC}$, and stacking of type $\mathrm{FCC}_{2}$ of CBA. What direction is indexed by 1 or 2 is completely arbitrary, although it is insightfull to realize that for perfect FCC the stacking direction is identical 

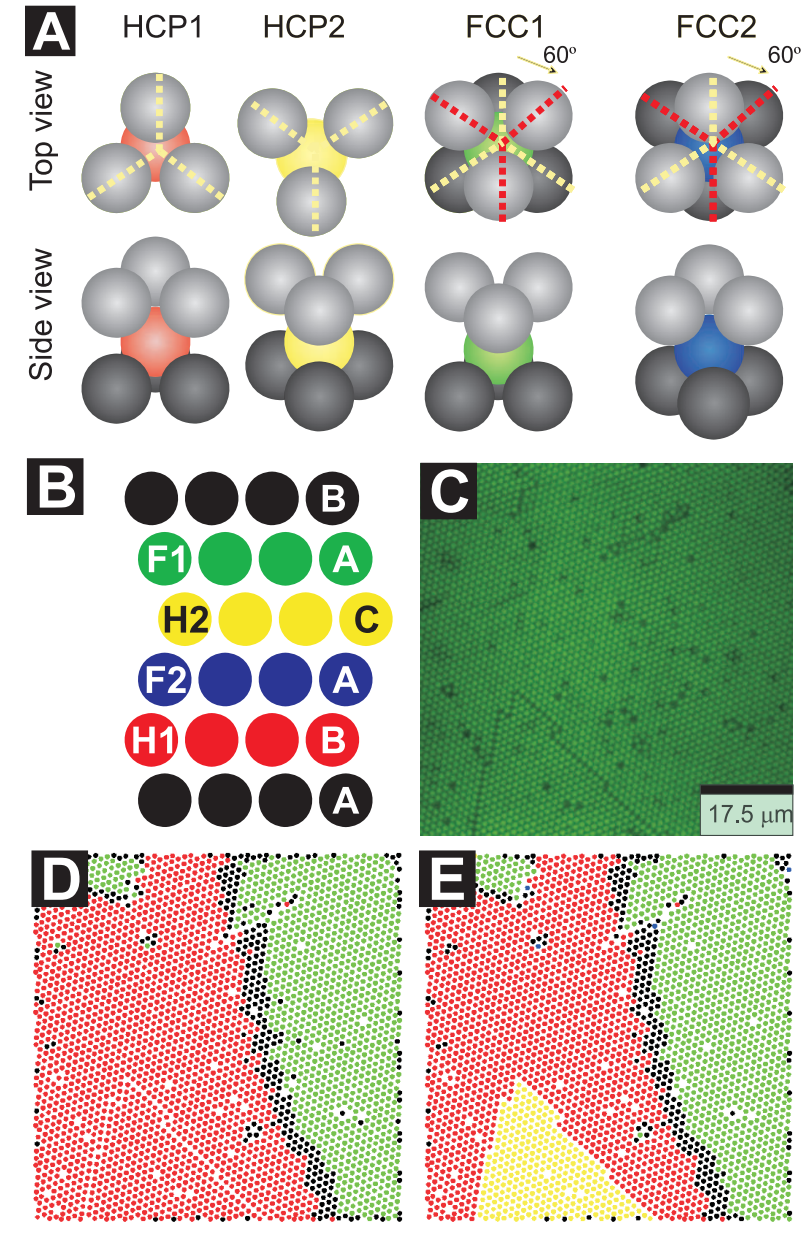

Fig. 2: (Colour on-line) Identification of stacking islands. A) Illustration of stacking type and stacking direction. The relative rotation of the particles in layers $L+1$ and $L-1$ is $60^{\circ}$ for $\mathrm{FCC}$ and $0^{\circ}$ for HCP. The change of stacking direction is characterised by a rotation of $180^{\circ}$ around the direction perpendicular to the hexagonal plane, resulting in a shift from $\mathrm{HCP}_{1}$ to $\mathrm{HCP}_{2}$ or from $\mathrm{FCC}_{1}$ to $\mathrm{FCC}_{2}$. B) Illustration of the stacking directions (side view). The different stacking directions are marked: $\mathrm{FCC}_{1}(\mathrm{~F} 1), \mathrm{FCC}_{2}(\mathrm{~F} 2), \mathrm{HCP}_{1}(\mathrm{H} 1)$ and $\mathrm{HCP}_{2}(\mathrm{H} 2)$. The lateral positions $\mathrm{A}, \mathrm{B}$ and $\mathrm{C}$ are shown as well. C) Confocal image with both a line-defect (left bottom corner) and a dislocation (hard to see in the image itself, but it runs through the image on the right side). D) The $\left|\psi_{3}\right|$ method results in identification of the HCP (red circles) and FCC (green circles) islands, but neighbouring islands with identical stacking type are identified as one island. Black symbols $(\bullet)$ indicate particles for which no stacking is assigned. E) The incorporation of the argument of $\psi_{3}$ results in a better definition of a stacking island, since the stacking direction is involved. (green circles) $\mathrm{FCC}_{1}$, (red circles) $\mathrm{HCP}_{1}$, (yellow circles) $\mathrm{HCP}_{2}$, (black circles) no stacking assigned. No $\mathrm{FCC}_{2}$ particles are present in this particular image. The partial dislocation clearly shows up in the wide area with black particles in panel (D) end (E).

throughout the system, whereas for perfect HCP the orientation alternates per layer. The argument of $\psi_{3}^{+} \pm \psi_{3}^{-}$ can distinguish between the stacking directions of $\mathrm{HCP}$ and FCC. $\psi_{3}^{+}+\psi_{3}^{-}$results in peaks separated by $\pi$ for the distinct $\mathrm{HCP}_{1}$ and $\mathrm{HCP}_{2}$ directions, as $\psi_{3}^{+}-\psi_{3}^{-}$does for $\mathrm{FCC}_{1}$ and $\mathrm{FCC}_{2}$. For analysis, we additionally use the constraints that either $\left|\psi_{3}^{+}+\psi_{3}^{-}\right|(\mathrm{HCP})$ or $\left|\psi_{3}^{+}-\psi_{3}^{-}\right|$ (FCC) should be 1.6 or larger, based on at least 1 particle in both the layer below and the layer above. For island size, a minimum island size of 10 particles was employed to reduce the noise. For samples TTC6 and De13, small islands at the border of the image were excluded from analysis since these are probably part of larger islands. In fig. 2C, D and $\mathrm{E}$ we illustrate the distinction between islands based on $\arg \left(\psi_{3}^{+} \pm \psi_{3}^{-}\right)$. In fig. $2 \mathrm{C}$ the confocal image is shown: a line-defect is clearly present. In fig. 2D the distinction between HCP and FCC based on the $\mid \psi_{3}^{+} \pm$ $\psi_{3}^{-} \mid$method gives HCP on both sides of the line-defects. With the $\arg \left(\psi_{3}^{+} \pm \psi_{3}^{-}\right)$method however, the different stacking directions of $\mathrm{HCP}_{1}$ and $\mathrm{HCP}_{2}$ can clearly be distinguished in fig. 2E. The method is limited to grains with 111 planes parallel to the coverglass: no criterion to extract the 3D grain orientation is included.

Before we proceed we should point out the two important distinctions between stacking islands and lateral islands. First of all, a single line-defect separates two lateral islands, but causes stacking islands in the layer above and the layer below as well [21]. Stacking islands should therefore be significantly smaller than lateral islands. Similar arguments apply for a slowly changing lateral position, but in those cases the border between stacking orientations is broad and does not result in problems for identification, which can easily be deducted from fig. 2E. Furthermore, the distinction based on stacking orientation does not exclude all islands next to line-defects from having an identical stacking orientation assignment: for line-defects present in three consecutive layers [21], identical stacking is assigned to particles on either side of the middle line-defect. We will relate lateral island dimensions $D_{\text {lat }}$ and stacking island dimensions $D_{\mathrm{FCC}}$ and $D_{\mathrm{HCP}}$ as a function of crystal grain dimension $D_{\mathrm{gr}}$ by simulations later on. We define the island dimension as the square root of the number of particles within it (either lateral or stacking) and the grain dimension $D_{\text {gr }}$ as the square root of the average number of particles per layer of a grain.

Identification of grains. To obtain good statistics of the island dimension as a function of $D_{\mathrm{gr}}$, particles within a single grain can be isolated, using the argument of the local bond order parameter $\psi_{6}$ :

$$
\psi_{6}\left(r_{j}\right)=\frac{1}{N} \sum_{k=1}^{N} \exp \left[6 i \theta\left(\mathbf{r}_{j k}\right)\right],
$$

where the summation $k$ runs over all, in total $N$, nearest neighbours of particle $j . \theta\left(\mathbf{r}_{j k}\right)$ is the angle between the bond vector $\mathbf{r}_{j k}$ between particles $j$ and $k$ and an arbitrary fixed reference axis. The absolute value of $\psi_{6}$ represents its crystallinity; the argument of $\psi_{6}$ represents a crystalline particle's orientation. The latter can be used to isolate grains, since grain boundaries are characterised 


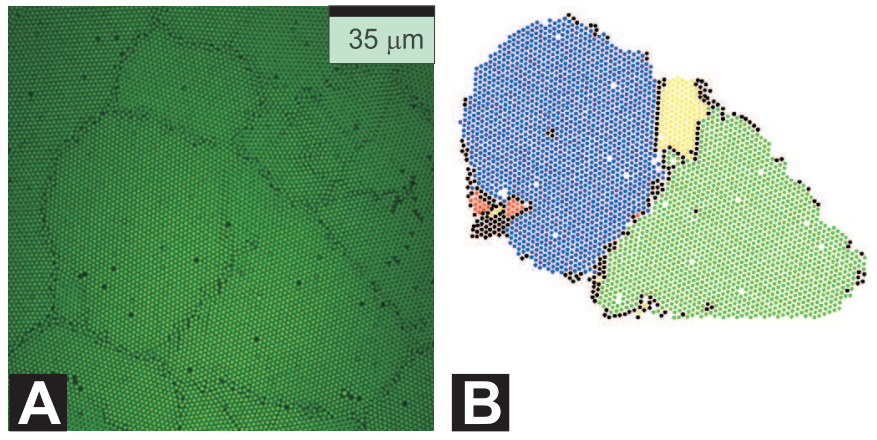

Fig. 3: (Colour on-line) Grains sizes and stacking islands. A) Confocal image of a crystal with multiple grains present. B) Stacking assignment of particles using the $\psi_{3}^{ \pm}$method, the particles in the grain are isolated using the argument of $\psi_{6}$. (green, blue circles) FCC; (red, yellow circles) HCP; (black circles) no stacking assigned.

by an abrupt change in crystal orientation. Since defects typically have a slightly different $\psi_{6}$ argument, an additional procedure is used to include all particles within the contours of the obtained grain. Defect particles outside of the grain which coincidentally have the same $\psi_{6}$ argument are excluded based on a next-neighbour criterion. The isolation works quite well, as shown in fig. 3 .

Simulations. - On-lattice Monte Carlo (MC) simulations were performed using a layer-by-layer nucleation and growth model. To simplify simulations, we used a single lattice, which is the same for $\mathrm{A}, \mathrm{B}$ and $\mathrm{C}$ lateral positions. Instead, the distinction between different stacking position was performed by assigning a corresponding label (equal to 0,1 or 2 ) to every occupied site $j$. This simplification might affect the structure at the island boundary, but was expected to reasonably reproduce the island structure. For more effective computation a list of free places was used, from which a random position $j$ was selected at every elementary MC step. Then either a nucleation event, with a certain probability $r_{n}$ or a growth attempt was performed. In all results presented here the nucleation rate $r_{n}=2 \times 10^{-5}$ was used, which yields island sizes comparable to that in the experiment. The nucleation step had $100 \%$ acceptance and the position label was chosen such that the creation of the FCC environment had a chance of $\alpha_{\mathrm{N}}$. A growth step at position $j$ was accepted if a randomly chosen neighbouring site $k$ was occupied. The position label was then assigned to be the same as at the site $k$. In addition, the growth step was rejected if the position label for the new particle was equal to the lateral position in the layer below. Periodic boundary conditions (PBC) were incorporated for bulk models, however these were switched off to simulate the finite-size effects. The results of the simulations were averaged over $N_{\text {av }}$ realizations (typically, $N_{\mathrm{av}}=100$ ).

\section{Results and discussion.}

Bulk vs. finite size. Figure $4 \mathrm{~A}$ shows a typical structure of a layer simulated with PBC. Since we do not
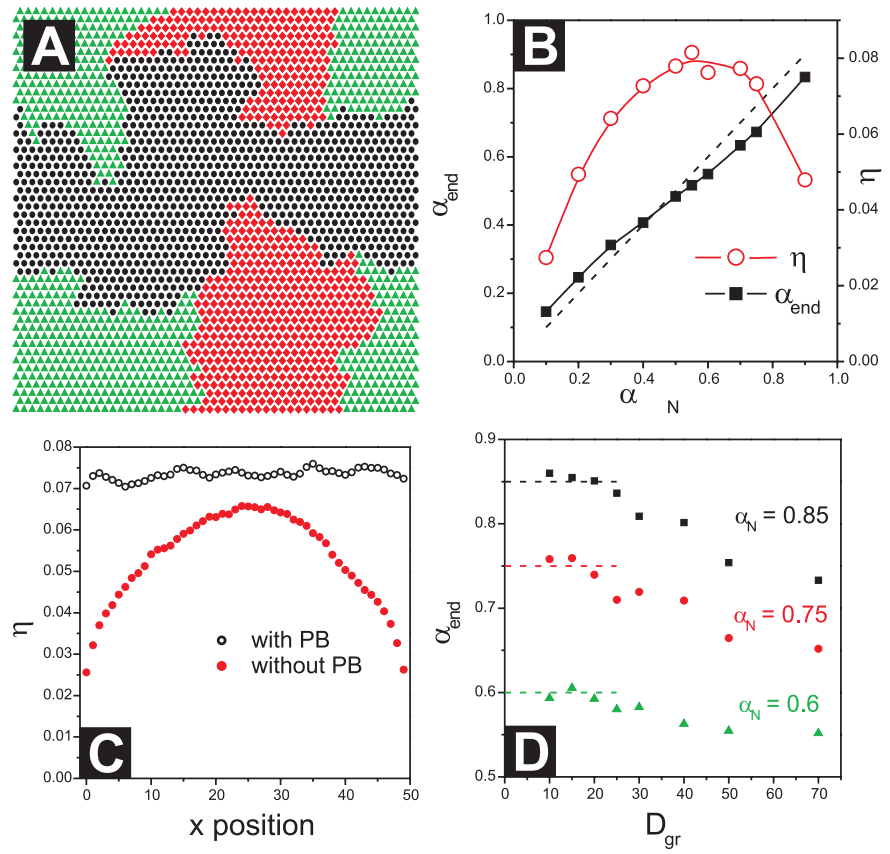

Fig. 4: (Colour on-line) Simulation results for the bulk (A, B) and small grains (C, D). A) A typical example of island configuration in the bulk obtained with $\mathrm{PBC}$ for a layer containing $50 \times 50$ sites. Different symbols are used for particles with different positional label. B) Dependence of the resulting stacking probability $\alpha_{\text {end }}(\boldsymbol{\square}$, left axis) and the defect density $\eta$ (०, right axis) on $\alpha_{\mathrm{N}}$. Distribution of the defect particles along the horizontal $x$-axis (averaged along the vertical $y$-direction) with (o) and without (•) PBC. D) The resulting stacking probability $\alpha_{\text {end }}$ as a function of the grain size for three values of $\alpha_{N}$. The horizontal dashed lines mark the $\alpha_{\mathrm{N}}$ values.

include a possibility for structure re-organisation in our model, the boundaries between the islands appear rough. The fraction $\alpha_{\text {end }}$ of the FCC stacking in the resulting structure (fig. $4 \mathrm{~B}$ ) is found to deviate from $\alpha_{\mathrm{N}}$, especially for the smallest and the largest values of $\alpha_{\mathrm{N}}$ ). This effect is related to the fact that an island (e.g. with $\mathrm{C}$ positions) can grow over a line-defect in the layer below (e.g., between $\mathrm{A}$ and $\mathrm{B}$ islands). This changes the stacking environment and, as a result, $\alpha_{\text {end }}$ shifts closer to $\approx 0.5$. Particles, which have at least one in-plane neigbour with a different position, are defined as defect particles. The density $\eta$ of such defect particles is found to display a maximum around $\alpha_{\mathrm{N}} \approx 0.5$ (fig. $4 \mathrm{~B}$ ). It decreases towards the extreme values, where the new islands are predominantly nucleated with the same stacking sequence and, therefore, have larger chance to merge without forming a line-defect.

Switching off PBC significantly affects the total number of defects and the positional distribution of defects (fig. 4C). Near the grain boundary the defects are depleted. Also the value of $\alpha_{\text {end }}$ slightly changes for the small grains (fig. 4D). When the grain size is smaller than the typical island size in the bulk, most layers consist of single islands and the resulting value of $\alpha_{\text {end }}$ is 

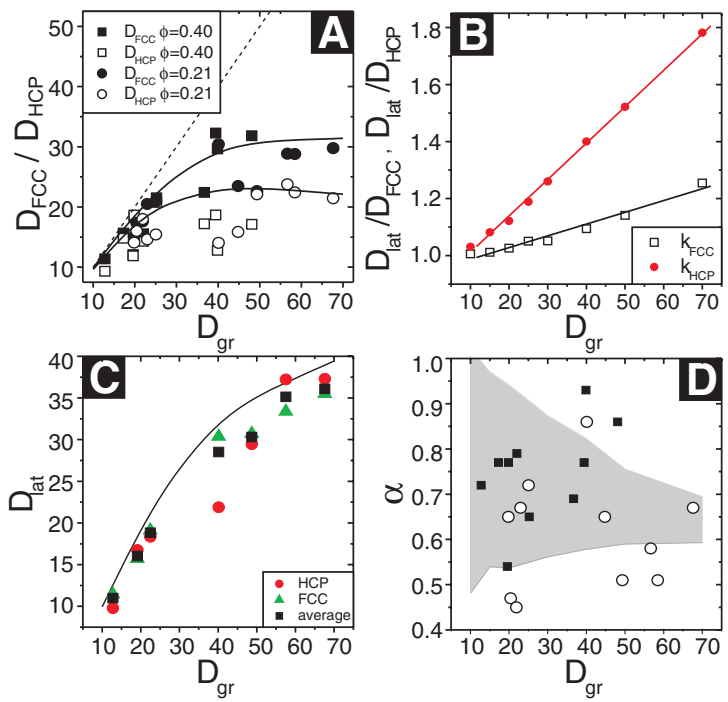

Fig. 5: (Colour on-line) Grain size effects. A) The stacking island dimensions as a function of grain dimension; experimental FCC island dimension (filled points), experimental HCP island dimension (open points) and modelled FCC and HCP island dimension (lines). The squares refer to sample De14 $\left(\phi_{0}=0.2\right)$ and the circles to sample De10 $\left(\phi_{0}=0.4\right)$. B) The prefactors $k_{\mathrm{FCC}}$ and $k_{\mathrm{HCP}}$ as a function of grain dimension $D_{\mathrm{gr}}$. C) The estimated lateral island dimension based on experimentally determined HCP stacking islands (circles), FCC islands (triangles) and the weight-average value (squares) as function of grain dimension. The line presents the simulations results. D) The fraction of FCC stacked particles as a function of grain dimension for experimental data (points) and model data (grey shaded) for $\alpha_{\mathrm{N}}=0.75$, with grey area $=2 \times$ standard deviation $\sigma$. $\mathbf{\square}$ : sample De14; ○: sample De10.

just determined by stacking probability $\alpha_{\mathrm{N}}$ used in the nucleation step. Note that, in experiment, strain at the grain boundary might enhance the number of defects.

Stacking islands vs. grain size. We measured the stacking island dimensions of several grains in our samples and obtained by simulation. For our experiments, we used the $\psi_{3}^{ \pm}$method to identify the stacking direction, with at least 20 crystal layers per grain. The first two layers contain more defects, which can be shown by measuring the amount of particles next to line-defects [21] per layer. Excluding the first two layers did not affect the measured island dimensions. The nucleation probability for FCC $\alpha_{\mathrm{N}}$ was 0.75 for the simulations, typically resulting in $\alpha_{\text {end }}=0.7$ (fig. 4D), similar to our experiments. In both cases, the island size initially rises with grain size (fig. 5A). Above $D_{\mathrm{gr}} \approx 30$, the stacking dimension only increases very slowly (fig. $5 \mathrm{~A}$ ) which means the number of islands quickly rises. $D_{\mathrm{FCC}}$ is usually significantly larger than $D_{\mathrm{HCP}}$. Because relatively more FCC islands have nucleated within a layer, these FCC islands should more frequently combine to form larger islands than the HCP islands, which explains the observed difference in island sizes. In general the variety of island sizes found in the
Table 1: The relationship between the size of stacking and lateral islands.

\begin{tabular}{lll}
\hline$\alpha_{\mathrm{N}}$ & $k_{\mathrm{FCC}}$ & $k_{\mathrm{HCP}}$ \\
\hline 0.6 & $0.93+0.0064 D_{\mathrm{gr}}$ & $0.90+0.0103 D_{\mathrm{gr}}$ \\
0.75 & $0.95+0.0041 D_{\mathrm{gr}}$ & $0.88+0.0128 D_{\mathrm{gr}}$ \\
0.85 & $0.96+0.0028 D_{\mathrm{gr}}$ & $0.84+0.0159 D_{\mathrm{gr}}$ \\
\hline
\end{tabular}

crystal increases with grain size. In small grains, linedefects usually run across grains. These defects are still present in larger crystals, but usually near the borders of the grain, with a sharp kink in it, such as in the left bottom in fig. 2C. Figure 5A contains datapoints of samples with $\phi_{0}=0.2(\mathrm{De} 10)$ and $0.4(\mathrm{De} 14)$, and sample history clearly does not play a (large) role in determining the typical island size. The higher viscosity of the De14 sample slows down the particle flux by sedimentation, apparently leading to similar island nucleation and growth mechanisms.

Lateral islands vs. stacking islands. We determined the relation between lateral islands and stacking islands by simulation for $\alpha_{\mathrm{N}}=0.75$. The lateral islands were identified by procedures similar to those used to identify stacking islands, but now using the lateral position instead of the stacking to identify the island. We defined $k_{\mathrm{FCC}}=$ $D_{\text {lat }} / D_{\mathrm{FCC}}$ and $k_{\mathrm{HCP}}=D_{\text {lat }} / D_{\mathrm{HCP}}$, which are plotted in fig. 5B against $D_{\mathrm{gr}}$. In the range of interest these relationships are approximately linear. For the smallest $D_{\text {gr }}$ most crystal planes are perfect and $k_{\mathrm{FCC}}=k_{\mathrm{HCP}}=1$. For much larger $D_{\mathrm{gr}}>150$ (not shown) $k_{\mathrm{FCC}}$ and $k_{\mathrm{HCP}}$ plateau.

Using these relationships, one can determine the size $D_{\text {lat }}$ of lateral islands out of the experimental found stacking islands dimensions as shown in fig. 5C. The evaluation is separately performed on the basis of $D_{\mathrm{FCC}}$ and $D_{\mathrm{HCP}}$. Using these results, the weight-average value of $D_{\text {lat }}$ is also evaluated using the relative fractions of the islands of both type as the weight factor.

FCC fraction. The overall stacking parameter $\alpha$ varies widely among the experimental grains (fig. 5D), but is on average $\sim 0.7$. The large fluctuation of $\alpha$ is related to the limited amount of particles in small grains. In our simulations, $\alpha_{\text {end }}$ was determined for $N_{\text {av }}=100$ grains. Most experimental data points lie within the grey area in fig. 5D, which represents the average $\alpha_{\text {end }} \pm 2 \sigma$ from simulations, with $\sigma$ the standard deviation.

To investigate the effect of $\alpha$ on the island dimension, we additionally determined $k_{\mathrm{HCP}}$ and $k_{\mathrm{FCC}}$ by simulation for $\alpha_{\mathrm{N}}=0.6$ and 0.85 . For the studied $\alpha_{\mathrm{N}}$ the relationships are listed in table 1. $\alpha$ has a strong effect for these $k$-functions, resulting in significantly higher multiplication factors for $\mathrm{HCP}$ at higher $\alpha$ (as long as $\alpha$ is larger than $\sim 0.5$ ). The dependence of $D_{\mathrm{FCC}}$ on $\alpha$ is much less strong. Our results suggest that this dependency should be reversed when $\alpha<0.5$.

The effect of $\alpha$ on the typical island dimensions was experimentally verified by measuring island sizes within 
Table 2: $D_{\mathrm{FCC}}$ and $D_{\mathrm{HCP}}$ in TTC and De.

\begin{tabular}{lll}
\hline Sample & $D_{\mathrm{FCC}}$ & $D_{\mathrm{HCP}}$ \\
\hline Decalin & $26 \pm 16$ & $16 \pm 12$ \\
TTC & $16 \pm 12$ & $13 \pm 9$ \\
\hline
\end{tabular}

several grains in TTC and De solvent. In TTC, $\alpha$ is $\sim 0.6$, slightly lower than in De, where $\alpha \sim 0.7$. The crystals studied have estimated grain dimensions of 70-100 diameters, a regime where the typical stacking island size is not expected to change very much anymore with grain size (fig. 5A). Since the image dimensions are identical for all measurements, we can compare the island dimensions found, even though part of the grain is not imaged (the lattice constants differ by $\sim 3 \%$ ). The results are summarized in table 2. Both stacking island dimensions are smaller in TTC, which should be related to the kinetics of island nucleation and growth. In TTC, the density of the solvent is nearly matched to that of the particles, resulting in a slower flux of particles, and therefore to more nucleation events, resulting in smaller stacking island dimensions. In De, the distribution of island sizes is very broad, but FCC islands are clearly larger than HCP islands. In TTC, both island sizes have decreased, but HCP and FCC are much more similar, clearly supporting the observations from our simulations: The recombination of stacking islands with identical orientation indeed seems to lead to larger FCC islands at higher $\alpha$.

Conclusion. - We investigated the effect of grain size and of the fraction $\alpha$ of FCC particles on the size of the islands with lateral $\mathrm{A}, \mathrm{B}$ or $\mathrm{C}$ position in RHCP sedimented hard-sphere crystals by confocal microscopy and by Monte Carlo simulations. The islands were identified by assigning a stacking (HCP or FCC) and a stacking direction ( 1 or 2 ) to every particle. The dimensions of the stacking islands were correlated (domains with identical stacking) to lateral island dimensions (islands with identical lateral positions $\mathrm{A}, \mathrm{B}$ or $\mathrm{C}$ ). The lateral islands are larger than the stacking islands, since the stacking islands depend on lateral positions in three layers, compared to one layer for stacking islands. In small grains $\left(D_{\mathrm{gr}}<\sim 30\right)$, the grains mainly consist of single stacking islands. In larger grains the amount of islands quickly rises and the island size seems to plateau. For $\alpha>0.5$, FCC islands are significantly larger than HCP islands, as $\alpha$ approaches 0.5 , the islands become smaller. This is related to the chance of finding an adjacent nucleated island with the identical stacking, which is lowest when $\alpha \sim 0.5$.

The authors acknowledge valuable discussions with H. LEKKERKERKER, P. SCHALl, A. LEFERINK OP REININK and R. Dullens. The work of VWAdV is part of the research program of the "Stichting voor Fundamenteel Onderzoek der Materie (FOM)", which is financially supported by the "Nederlandse Organisatie voor Wetenschappelijk Onderzoek (NWO)". Support of VWAdV by the DFG through the SFB TR6 is acknowledged. Schlumberger is acknowledged for support of JMM.

\section{REFERENCES}

[1] Wu X., Zhu Y. T., Chen M. W. and Ma E., Scr. Mater., 54 (2006) 1685.

[2] Collier C. P., Saykally R. J., Shiang J. J., Henrichs S. E. and Heath J. R., Science, 277 (1997) 1978.

[3] Gilbert B., Huang F., Zhang H., Waychunas G. A. and BANFiELd J. F., Science, 305 (2004) 651.

[4] Puntes V. F., Krishnan K. M. and Alivisatos A. P., Science, 291 (2001) 2115.

[5] Pusey P. N. and van Megen W., Nature, 320 (1986) 340.

[6] Gasser U., Weeks E. R., Schofield A., Pusey P. N. and Weitz D. A., Science, 292 (2001) 258.

[7] de Villeneuve V. W. A., Dullens R. P. A., Aarts D. G. A. L., Groeneveld E., Scherff J. H., Kegel W. K. and LeKKERKERKER H. N. W., Science, 309 (2005) 1231.

[8] Hoogenboom J. P., van Langen-Suurling A. K., Romijn J. and van BlaAderen A., Phys. Rev. E, 69 (2004) 051602.

[9] Solomon T. and Solomon M. J., J. Chem. Phys., 124 (2006) 134905.

[10] Bolhuis P. G., Frenkel D., Mau S. C. and Huse D. A., Nature, 388 (1997) 235.

[11] Bruce A. D., Wilding N. B. and Ackland G. J., Phys. Rev. Lett., 79 (1997) 3002.

[12] Mau S. C. and Huse D. A., Phys. Rev. E, 59 (1999) 4396.

[13] Pusey P. N., van Megen W., Bartlett P., Ackerson B. J., Rarity J. G. and Underwood S. M., Phys. Rev. Lett., 63 (1989) 1989.

[14] Zhu J., Li M., Rogers R., Meyer W., Ottewill R. H., Crew S.-S. S., Russel W. B. and Chaikin P. M., Nature, 387 (1997) 883.

[15] Kegel W. K. and Dhont J. K. G., J. Chem. Phys., 112 (2000) 12.

[16] Petukhov A. V., Dolbnya I. P., Aarts D. G. A. L., Vroege G. J. and Lekkerkerker H. N. W., Phys. Rev. Lett., 90 (2003) 028304.

[17] Hoogenboom J. P., Derks D., Vergeer P. and van Blaaderen A., J. Chem. Phys., 117 (2002) 11320.

[18] Dolbnya I. P., Petukhov A. V., Aarts D. G. A. L., Vroege G. J. and LeKKerkerker H. N. W., Europhys. Lett., 72 (2005) 962.

[19] Pronk S. and Frenkel D., J. Chem. Phys., 110 (1999) 4589.

[20] Auer S. and Frenkel D., Nature, 409 (2001) 1020.

[21] Meijer J. M., de Villeneuve V. W. A. and Petukhov A. V., Langmuir, 23 (2007) 3554.

[22] Bosma G., Pathmamanoharan C., De Hoog E. H. A., Kegel W. K., van BlaAderen A. and LeKkerkerker H. N. W., J. Colloid Interface Sci., 245 (2002) 292.

[23] Crocker J. C. and Grier D. G., J. Colloid Interface Sci., 179 (1996) 298.

[24] Dullens R. P. A. and Petukhov A. V., Europhys. Lett., 77 (2007) 58003. 